\title{
Initial experience of laparoscopic right lobectomy after portal vein embolization
}

\author{
Hwui-Dong CHO, Ki-Hun KIM* \\ Division of Hepatobiliary Surgery and Liver Transplantation, Department of Surgery, Asan Medical Center, \\ University of Ulsan College of Medicine, Seoul, Korea
}

Introduction: Portal vein embolization is a technique used before hepatic resection to increase the size of remnant liver after surgery. This therapy redirects portal blood to segments of the future liver remnant, resulting in hypertrophy. The purpose of this study was to find out the safety and feasibility of laparoscopic right lobectomy after portal vein embolization.

Methods: All consecutive cases of laparoscopic right lobectomy after portal vein embolization between July 2014 and April 2018 in a tertiary referral hospital were enrolled in this retrospective cohort study. All surgical procedures were performed by one surgeon. There were 14 cases of laparoscopic right lobectomy after portal vein embolization. The group was analysed in terms of donor demographics, preoperative data, postoperative outcomes.

Results: The mean age of the donors was $51.3 \pm 6.7$ years, the gender ratio for men and women was 18: 96 . The mean operative time was $402.5 \pm 78.8$ minutes and mean postoperative hospital stay was $10.1 \pm 1.7$ days. The number of complications was 6 cases (5.3\%) and among them, the Clavien-Dindo classification III or higher complication was 2 (1.8\%). There were no mortality cases.

Conclusions: Minimal invasive living donor right hepatectomy was a safe and feasible procedure for donors. It showed an acceptable incidence of complications. The authors suggest that minimal invasive living donor right hepatectomy could be a reasonable operative option for donors in terms of cosmetic effect. 